\title{
AVALIAÇÃO DO DESEMPENHO DE POLÍMEROS NATURAIS MODIFICADOS NO AUMENTO DA CAPACIDADE DE CAMPO DE SOLOS ARENOSOS
}

\author{
Adriano A. A. Neto ${ }^{1 *}$, Denise F. S. Petri ${ }^{1}$ \\ 1 -Instituto de Química, Universidade de São Paulo (USP), São Paulo, SP
}

adriano.neto@usp.br

\begin{abstract}
Resumo: Polímeros naturais modificados foram avaliados em termos de desempenho na capacidade de campo de solos arenosos. As variações realizadas a nível molecular se traduzem em menores perdas de água por percolação ou evapotranspiração. Ensaios foram conduzidos em laboratório variando-se as propriedades físico-químicas dos polímeros naturais, que se mostraram efetivos ao aumentarem a retenção de água do solo, se posicionando como uma alternativa promissora e de perfil ecotoxicológico superior para uso em solos acometidos por intenso estresse hídrico, permitindo a utilização econômica de áreas outrora desafiadoras mesmo não agricultáveis.
\end{abstract}

Palavras-chave: polimero natural, solo arenoso, capacidade de campo, ponto permanente de murcha, retenção, água

Evaluation of Natural Modified Polymers performance on increasing field capacity of sandy soils

\begin{abstract}
Modified natural polymers have been evaluated in terms of performance on increasing field capacity of sandy soils. The changes performed at the molecular level have been shown effective on reducing water loss by percolation or evapotranspiration. The study carried out in the lab scale has been conducted varying natural polymers' physicalchemical properties, which proved to be effective by increasing hydric retention in soils. These polymers seem to be a promising and superior alternative for soils affected by intense hydric stress, allowing economical usage of challenging areas or even areas where agriculture was not viable.
\end{abstract}

Keywords: natural polymer, sandy soil, field capacity, wilting point, water, retention

\section{Introdução}

Soluções capazes de atuar no solo de maneira a aumentar a disponibilidade de água para plantas possuem um promissor campo de aplicação na agricultura. Neste sentido, define-se alguns conceitos relevantes onde estas soluções podem atuar, entre eles a capacidade de campo e o ponto permanente de murcha. A capacidade de campo é definida como a quantidade máxima de água disponível após 
drenagem da água, por ação gravitacional, através de percolação entre os macroporos do solo. ${ }^{1}$ Já o ponto de murcha permanente representa o limite inferior de água disponível para as plantas no solo - a partir de certo nível de umidade a planta não é capaz de obter água do solo em quantidades suficientes para suprir a demanda para manutenção de sua viabilidade. ${ }^{2}$ Toda água existente entre a capacidade de retenção de campo e o ponto permanente de murcha representa a água que estará efetivamente disponível para a planta. Polímeros sintéticos com alta capacidade de adsorção de água, como por exemplo, poliacrilamidas (PAM) podem ser utilizados na agricultura com o objetivo de melhorar a retenção de água e melhorar o desenvolvimento de culturas agrícolas. Embora promissores em termos de funcionalidade, estes polímeros sintéticos não são biodegradáveis e seus produtos de degradação podem ser perigosos ao meio ambiente ${ }^{3}-$ a citar como exemplo monômeros de acrilamida, considerados potencialmente carcinogênicos. Polímeros naturais surgem como fortes candidatos para aplicações agrícolas devido à possibilidade de entregarem desempenho efetivo ao mesmo tempo que possuem um perfil eco toxicológico superior. O solo pode perder água através dos fenômenos de percolação, escoamento de superfície e evapotranspiração ${ }^{4}$ e, aditivos químicos biodegradáveis que possuam a capacidade de aumentar essa disponibilidade de água serão benéficos para a planta ao favorecer o desenvolvimento da cultura mesmo em condições de estresse hídrico. Macromoléculas de origem natural podem ser modificadas quimicamente com o objetivo de aumentar a capacidade de adsorção de água e apresentar um desempenho superior quando utilizadas na agricultura.

O objetivo deste trabalho é avaliar uma série de macromoléculas de origem natural, modificadas ou não, em relação a seu desempenho na capacidade de campo de solos arenosos e também no ponto de murcha, buscando determinar se há aumento na biodisponibilidade de água para a planta ao modificar o solo estudado.

\section{Experimental}

Determinação da capacidade de campo em solos arenosos tratados com polímeros naturais modificados

Os polímeros em estudo foram avaliados em termos da capacidade de campo conferida a solos arenosos tratados em concentrações de 100, 400, 700 e 1000 ppm. Os polímeros foram selecionados de modo a viabilizar um estudo comparativo entre as propriedades físicas e químicas dos materiais, 
notadamente massa molar e grau de substituição, e sua capacidade de gerar resposta na capacidade de retenção hídrica. A amostra de solo arenoso foi retirada da região de Piracicaba, estado de São Paulo e teve sua umidade determinada previamente por termogravimetria a partir da colocação em estufa a $105{ }^{\circ} \mathrm{C}$ até massa constante. Amostras de aproximadamente $15 \mathrm{~g}$ de solo foram tratadas com quantidades de polímeros de forma a alcançar a concentração em estudo (100, 400, 700 e 1000 ppm) e submetidas ao ensaio de capacidade de campo, em triplicata. A figura 1 mostra o aparato montado para a realização do ensaio de retenção: neste aparato o solo tratado, ao receber água simulando processo de irrigação ou precipitação - atinge a saturação e, logo em sequência, é deixado em repouso por aproximadamente 90 minutos, de maneira a drenar através de ação da gravidade, até que o equilíbrio seja atingido.

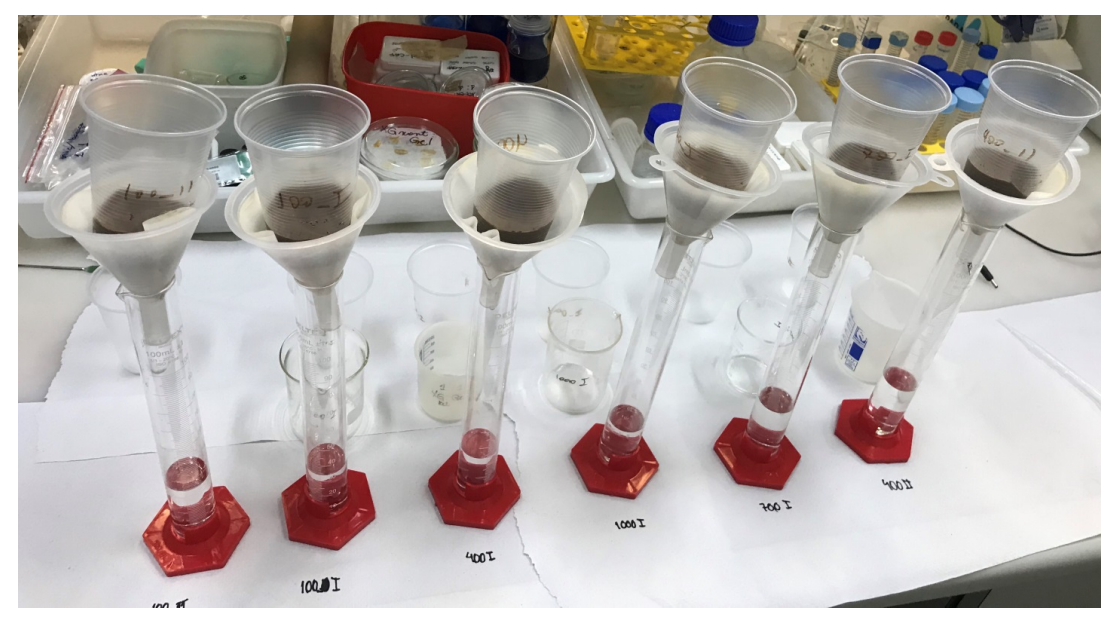

Figura 1 - Fotografia do aparato montado para realização dos ensaios de retenção hídrica e determinação da capacidade de campo.

Determinação de biodisponibilidade de água através da simulação do ponto de murcha permanente

Em seguida, a solução percolante coletada nas provetas é submetida à análise de condutividade (Condutivímetro Digimed DM-31) e $\mathrm{pH}$ (papel indicador de $\mathrm{pH}$ - Merck). Com o intuito de determinar quanto da água retida estará disponível para a planta até o ponto de murcha permanente, descrito na literatura como 15 bar, ${ }^{5}$ é utilizado um dinamômetro (Impac, modelo IP-90 DI, acoplado com célula de carga de até $500 \mathrm{~N})$ - pressões crescentes (1, 5 e 15 bar) são aplicadas no solo de 
maneira a simular progressão de estresse hídrico. Para garantir uniformidade de aplicação da pressão e fluxo de água para o meio externo, foi desenvolvido um cilindro de ABS (Acrilonitrila Butadieno Estireno) vazado em sua lateral inferior. Neste ensaio, parte da água é liberada e parte da água permanece retida na amostra, representando aquela água que não estaria disponível para a planta em situação real de campo.

\section{Resultados e Discussão}

Os ensaios foram realizados utilizando solo arenoso, rico em areia e que possui menor capacidade de retenção se comparado aos solos argilosos, ricos em matéria orgânica. O solo condicionado com os polímeros em estudo, a depender da concentração aplicada, tiveram sua capacidade de campo aumentada, indicando possível efeito benéfico para as plantas ali cultivadas. A análise dos dados foi realizada utilizando o programa Minitab 19, utilizando a ferramenta ANOVA, com intervalo de confiança de $95 \%$.

A figura 2 mostra os valores de capacidade de campo obtidos após o condicionamento do solo com o polímero 1. O polímero 1 não apresentou resposta em retenção estatisticamente diferente do controle à concentração de 100 ppm, porém a partir de 400 ppm foi possível observar efeito positivo em retenção hídrica, sendo este efeito diretamente proporcional ao aumento de concentração empregada, na faixa de concentração estudada.

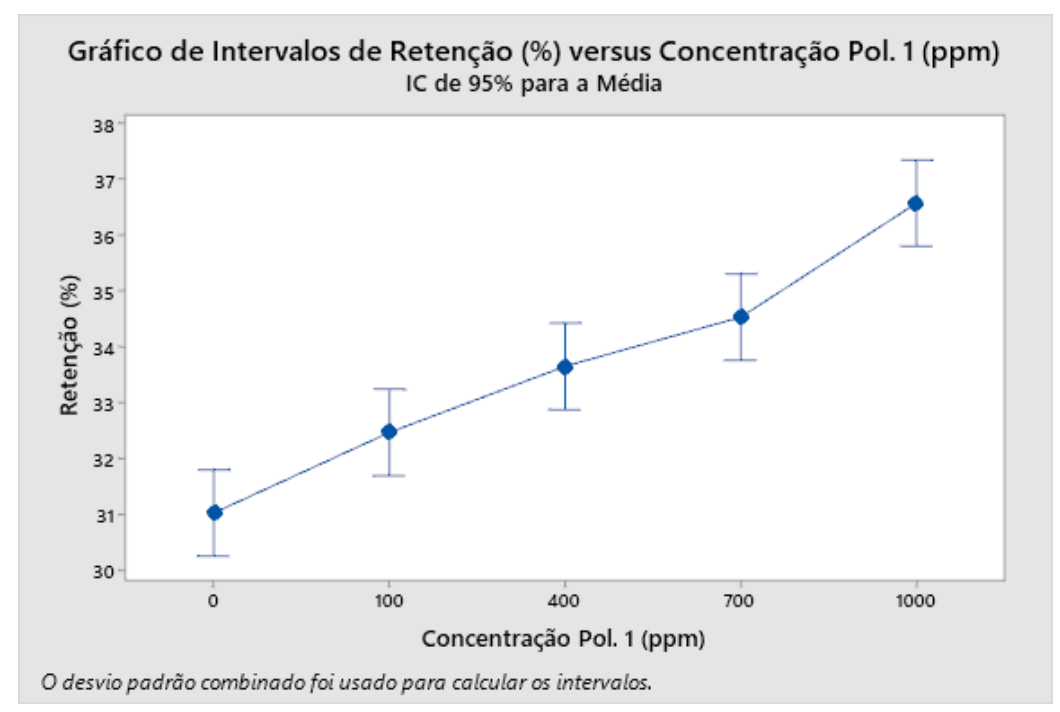

Figura 2 - Gráfico obtido para a capacidade de campo após utilização do polímero 1 em solo arenoso. 
A figura 3 mostra os valores de capacidade de retenção de campo para os solos tratados com o polímero 2. O polímero 2, por sua vez, apresenta retenção hídrica estatisticamente diferente do controle já a partir de 100 ppm, indicando que, a partir desta concentração já é possível obter efeito benéfico a partir de sua utilização - de maneira semelhante ao polímero 1, o aumento da concentração empregada no condicionamento da amostra de solo também oferece resposta diretamente proporcional em capacidade de retenção hídrica. O polímero 3, ao contrário dos demais estudados, não apresentou diferença estatisticamente relevante em relação ao controle em nenhuma das concentrações avaliadas, indicando que a adição deste aditivo químico não é capaz de aumentar a retenção hídrica do solo na faixa de estudo.

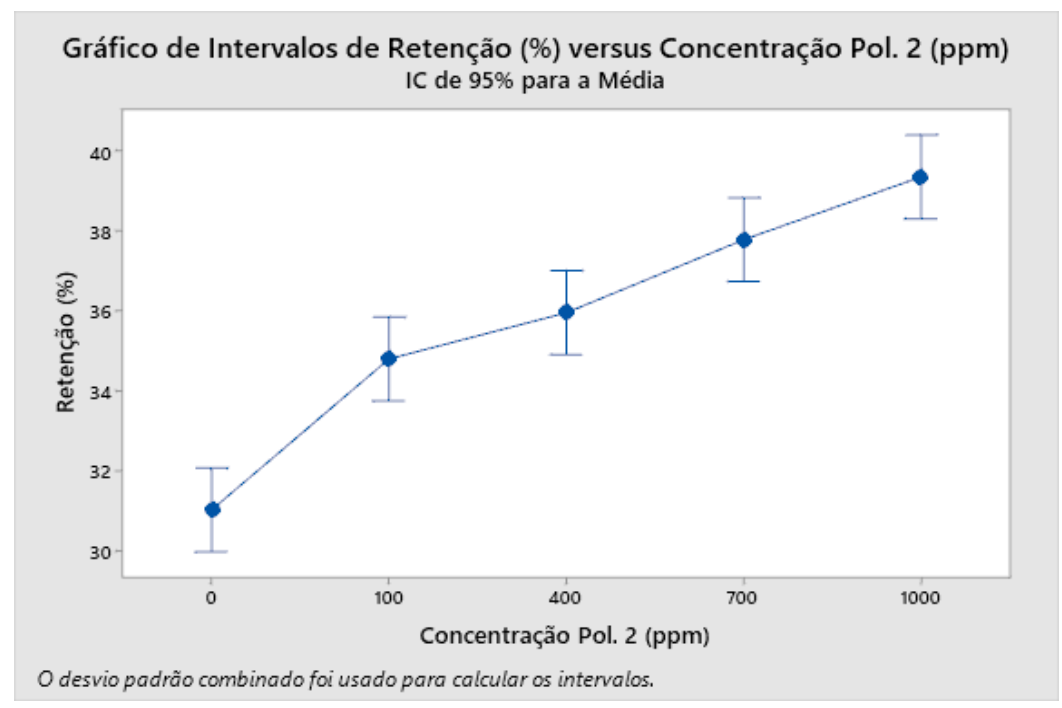

Figura 3 - Gráfico obtido para a capacidade de campo após utilização do polímero 2 em solo arenoso.

Por fim, após a obtenção dos gráficos foi realizada análise dos intervalos de confiança auferidos para os modelos estatisticamente relevantes, ou seja, aqueles modelos obtidos através do uso dos polímeros 1 e 2 - esta análise confirmou que não há, entre eles, sobreposição dos intervalos de confiança de $95 \%$ a partir de $400 \mathrm{ppm}$. Com isso é possível inferir que, ao passo que ambos estes polímeros 1 e 2 conferem ao solo aumento da capacidade de campo, a partir de 400 e 100 ppm, respectivamente; o polímero 2 apresenta desempenho destacadamente superior ao polímero 1 a partir de 400 ppm. 
Ao avaliar os resultados obitdos à luz das propriedades físicas e estrutura química dos polímeros estudados, é possível concluir que a massa molar parece ter uma relação direta com a capacidade de retenção hídrica dos solos - isso porque os polímeros 1 e 2 possuem massa molar $2 \cdot 10^{5}$ maior do que o polímero 3. É sabido que os polímeros naturais possuem, em sua grande parte, grupos polares covalentemente ligados à sua estrutura, sendo ricos em hidroxilas terminais. De fato, polímeros de alta massa molar podem apresentar conformação em forma de coil quando em solução aquosa, sendo estabilizados por interações intramoleculares e intermoleculares. ${ }^{6}$ Avaliando o cenário de aumento de retenção hídrica quando polímeros de alta massa molar são utilizados, acredita-se que a água pode na estabilização do coil através de interações intermoleculares do tipo interações de hidrogênio, o que gera um efeito de atração de água para o interior desta rede polimérica, o que se traduz, a um nível macro, na elevação da capacidade de campo em solos tratados. Enquanto o polímero 1 não apresenta modificação química, o polímero 2 foi modificado quimicamente de forma a apresentar interações intermoleculares mais fortes, sobretudo com as moléculas de água e essa é a hipótese que explica o porquê do polímero 2 apresentar desempenho superior quando comaparado ao 1, conforme resultados obtidos no ensaio de capacidade de campo. Agora, não só interações dipolo-dipolo e de hidrogênio estão disponíveis, mas também interações do tipo íondipolo, notadamente mais fortes - com isso, o efeito de atração de água para o interior da rede polimérica é mais pronunciado e mais água é retida no microambiente onde este polímero fora adicionado. Nota-se que, embora essa modificação química signifique maior impedimento estérico e, consequentemente, é esperado que afete a estabilidade do coil, o novo tipo de interação incorporado ao sistema é capaz de prevalecer e elevar a atração hídrica deste solo condicionado com o polímero 2. É de esperar também que, nutrientes hidrosolúveis possam ser carreados e retidos por essa rede polimérica e disponibilizados para a planta conforme sua necessidade através de sua estrutura radicular. O ensaio para determinação de biodisponibilidade de água não apresentou reprodutibilidade e resultados conclusivos - através do experimento realizado não foi possível simular com fidedgnidade a progressão do estresse hídrico no solo e, consequentemente, o ponto de murcha permanente não foi efetivamente alcançado. De fato, este método está sujeito a erros sobretudo decorrentes da falta de equilíbrio hidrostático da amostra quando submetido às pressões do estudo ${ }^{7}$ - entende-se que, a água disponível entre os macroporos do solo não é liberada com a mesma dinâmica em todas as faixas do estudo - isso significa que, o perfil de liberação de 
água é alterado de acordo com fatores além unicamente da quantidade água naquele solo - ao passo que a água na capacidade de campo é relativamente fácil de ser retirada do sistema, ao se aproximar do ponto de murcha permanente essa água fica retida mais fortemente ao solo, o que, na prática, acaba por inviabilizar a absorção de água pelo sistema radicular da planta e a leva à morte. Os valores obtidos para $\mathrm{pH}$ não variaram entre as soluções percoladas, ao passo que a condutividade aumentou conforme o aumento da concentração do polímero, não sendo possível traçar conclusões em termos de retenção de nutrientes a partir deste grupo de dados.

\section{Conclusões}

Um polímero natural modificado quimicamente apresentou um bom desempenho quanto utilizado para aumentar a capacidade de campo de solos arenosos. Além da massa molar polimérica, a força das ligações intermoleculares entre o polímero e a água se mostra relevante ao potencializar o efeito de retenção hídrica. Esse sistema proposto é promissor para aplicação em regiões suscetíveis a estresse hídrico de caráter contínuo e prolongado, possivelmente retardando a chegada do ponto de murcha permanente ao aumentar a capacidade de campo destes solos e retardar as perdas de umidade por percolação e evapotranspiração.

\section{Referências}

1. Borém. A. em Sugarcane: Agricultural Production, Bioenergy and Ethanol; Santos F.; Borém, A.; Caldas, C., eds.; Academic Press: Brazil, 2015, cap. 8.

2. https://www.infoteca.cnptia.embrapa.br/handle/doc/275940, acessada em: Outubro, 2020.

3. Thombare, N.; Mishra, S.; Siddiqui, M.Z.; Jha, U.; Singh, D. Mahajan, G. R.; Carbohydr. Polym. 2018, 185, 169-178.

4. https://www.nature.com/scitable/knowledge/library/soil-water-dynamics-103089121/, acessada em: Outubro 2020.

5. Yao, N.; Li, Y.; Xu, F.; Liu, J.; Chen, S.; Ma, H.; Chau, H. W.; Liu, D. L.; Li, M.; Feng, H. Agricultural Water Management, 2020, 229, 105954-105969.

6. Valle, L. J. ; Díaz, A.; Puiggalí, J..; Gels. 2017, 3, 27-55.

7. Solone, R.; Bitelli, M.; Tomei, F.; Morari, F; J. Hydrol. (Amsterdam, Neth.), 2012, 470-471, $65-$ 74. 
8. Yang, L.; Yang, Y.; Chen, Z.; Guo, C..; Li, S... Ecological Engineering, 2014, 62, 27-32.

9. Rabat, N. E.; Hashim, S.; Majid, R. A. Procedia Eng. 2016, 148, 201-207. 